\title{
Response function of the 2D quantum electron solid
}

To cite this article: S T Chui and B Tanatar 1995 J. Phys.: Condens. Matter 75865

View the article online for updates and enhancements.

\section{Related content}

- Dislocations in the melting of two-
$\frac{\text { dimensional electron crystals }}{\text { B Tanatar and S -T Chui }}$
- Dispersion Laws of Collective Excitations
$\frac{\text { in Crystalline and Superfluid }{ }^{4} \mathrm{He} \text { Related }}{\text { via Density-Functional Theory }}$
V. Tozzini and M. P. Tosi
- Self-consistent phonon and
$\frac{\text { magnetophonon and cubic anharmonic }}{\text { corrections of the 2D electron lattice }}$
K Esfariani and Siu Tat Chui

\section{Recent citations}

Phase diagram of the two-dimensional quantum electron freezing with external

impurities

S. T. Chui

\section{IOP ebooks}

Bringing you innovative digital publishing with leading voices to create your essential collection of books in STEM research. Start exploring the collection - download the first chapter of every title for free. 


\title{
Response function of the 2D quantum electron solid
}

\author{
S T Chui† and B Tanatarł \\ $\dagger$ Bartol Research Institute, University of Delaware, Newark, DE 19716, USA \\ $\ddagger$ Physics Department, Bilkent University, 06433 Ankara, Turkey
}

Received 28 December 1994, in final form 24 May 1995

\begin{abstract}
We study the static density response function of the 2D quantum electron solid with analytic and quantum Monte Carlo techniques. The 'longitudinal' response function at small and intermediate momentum transfer from the simulation is well approximated by the analytic results using phonons alone, provided the Debye-Waller factor is included. The 'transverse' response function and the large momentum transfer longitudinal response function from simulations are larger than the analytic results from contributions with phonons alone.
\end{abstract}

Recently there has been much interest in the low-density limit of two-dimensional (2D) electrons in GaAs heterojunctions in an external magnetic field [1,2], and Si-MOSFET in high and zero fields $[2,3]$, where a freezing transition to a solid seems to occur as the density is lowered. This motivated a systematic study of the 2D quantum electron solid. Physical measurements explore response functions. Typical responses are the particle-hole response, which is explored in shear modulus, conductivity and capacitance-type experiments, or single-particle responses, as in optical luminescence involving core holes and in tunnelling experiments. However, there has not been much theoretical study of the response in the solid state. The response of the solid is due to coupling of external perturbations to elementary excitations of the solid. The elementary excitations can be phonons [4] or defect waves [5]. In this paper we study a 'longitudinal' and a 'transverse' static density response function with quantum Monte Carlo calculations and compare them with the phonon contribution to the response function. We find that the 'longitudinal' response function at small and intermediate momentum transfer is well approximated by the analytic results using phonons alone (figure 1 $(a)-(c)$ ), provided the Debye-Waller factor is included. On the other hand, the 'transverse' and the large momentum transfer longitudinal response functions from the simulation are larger than that from the contributions with phonons alone (figure $2(a)$ and $(b)$ ). The difference of the response functions may be due to inadequacies of the anharmonic calculation or to contributions from defect waves. We now explain our results in detail.

The static density response function $\chi(q)$ can be related to the change in ground-state energy $\Delta E$ when an external static potential $v_{e x t}(r)=v_{q} \sin (q r)$ is applied:

$$
\Delta E=-0.25 \chi(q) v_{q}^{2} / \rho+O\left(v_{q}^{4}\right) \text {. }
$$

Here $\rho$ is the density of the system. To explain our terminology we first recapitulate the calculation for the response function of the solid. For a solid, the electrons are at positions $\boldsymbol{r}_{j}=\boldsymbol{r}_{j 0}+\delta \boldsymbol{r}_{j}$. For a wave vector $q$ along the $x$ direction the driving energy is

$$
\begin{array}{r}
\sum_{j} v_{q} \sin \left(q x_{j}\right)=\sum_{j} v_{q}\left[\sin \left(q x_{j 0}\right)+q \delta x_{j} \cos \left(q x_{j 0}\right)\right] \\
=v_{q}\left(\sum_{j} \sin \left(q x_{j 0}\right)+q\left(\delta x_{q}+\delta x_{-q}\right) / 2\right)
\end{array}
$$



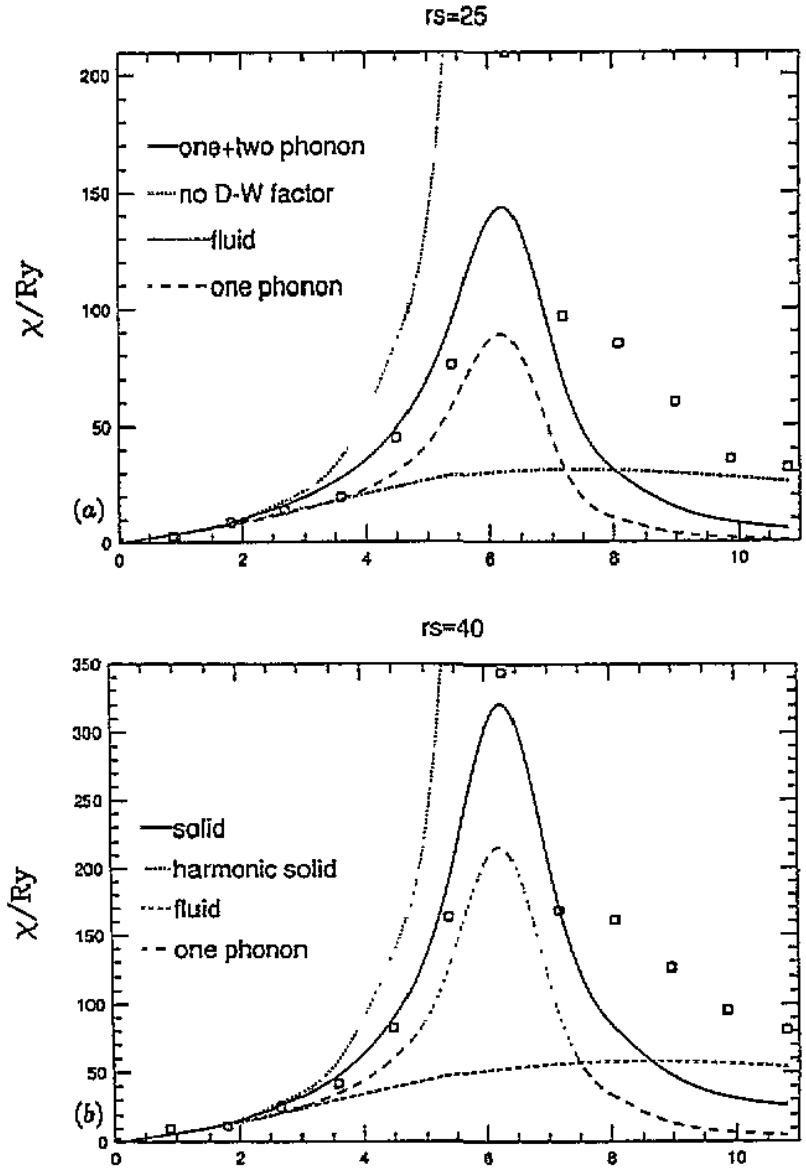

$\mathrm{rs}=75$

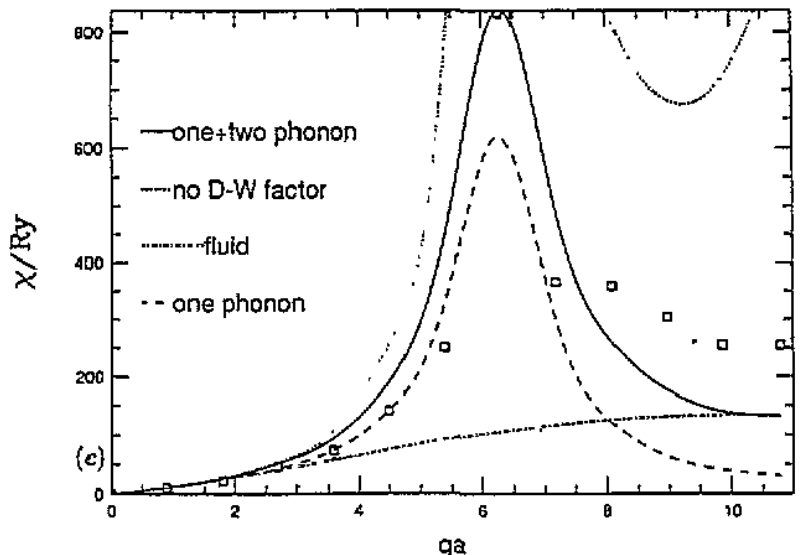

Figure 1. The 'longitudinal' response function $\chi(q, 0)$ in units of $\mathrm{Ryd}^{-1}$ as a function of the wavevector times the Iattice constant. The Monte Carlo results are indicated by the open squares. The analytic results for the one-phonon contribution with and without a Debye-Waller factor, the sum of the one- and two-phonon contributions and the fluid in the Hubbard approximation are shown by the broken, dotted, full and chain curves. Different densities for $r_{s}=25,40$ and 75 are shown in $(a)-(c)$. 
where

$$
\delta x_{q}=N^{-0.5} \sum_{j} \delta x_{j} \exp \left(\mathrm{i} q x_{j 0}\right)
$$

and the static response $\chi$ is given by

$$
\chi(q)=\sum_{n}|\langle n|\exp (\mathrm{i} q \cdot r)| 0\rangle|^{2} /\left(E_{n}-E_{0}\right) .
$$

The excited states $|n\rangle$ can be phonons or defect waves. If we include only those contributions from the phonons, we find that $\chi$ is approximately given by $\chi_{\text {phonon }}(q+K) \approx \chi_{1}+\chi_{2}$ where the one- and two-phonon contributions are given by

$$
\chi_{1}(q+K)=\left[(q+K) \cdot e_{q i}\right]^{2} \exp \llbracket-\left\{\left([(q+K) \cdot \delta r]^{2}\right\rangle\right\} \rrbracket / m \omega_{q i}^{2}
$$

and

$$
\begin{aligned}
\chi_{2}(q+K)= & 0.25\left(\hbar / m^{2}\right) \sum_{p}\left[(q+K) \cdot e_{p l}\right]^{2}\left[(q+K) \cdot e_{q-p, l^{\prime}}\right]^{2} \\
& \times \exp \left[-\left\{\left\{[(q+K) \cdot \delta r]^{2}\right\rangle\right\}\right] /\left[\omega_{p l} \omega_{q-p, l^{\prime}}\left(\omega_{p l}+\omega_{q-p, l^{\prime}}\right)\right] .
\end{aligned}
$$

For small $q$, because of the dot product, the longitudinal mode dominates; we get

$$
\chi_{\text {phonon }}(q) \approx q r_{s}^{2} a_{B} / 2 \mathrm{Ryd}^{-1} \text {. }
$$

We have evaluated $\chi$ for $r_{s}=25,40$ and 75 using the harmonic phonon frequencies [6]. Anharmonic corrections [4] to the longitudinal phonon frequencies at these densities are less than ten per cent. The analytical results for $\chi(q, 0)$ are shown in figures $1(a)-(c)$ by the full curve. (The two arguments in $\chi$ are meant to indicate the $x$ and $y$ components of $q$. This is to distinguish the Umklapp from the direct processes.) Also shown in figure 1 are the one-phonon contribution with and without the Debye-Waller factor and the fluid result in the Hubbard approximation [11]:

$$
\chi_{\text {fluid }}(q)=\chi_{0} /\left[1+(1-G(q)) v(q) \chi_{0}\right]
$$

where

$$
\chi_{0}=f\left(q / 2 k_{F}\right) / 2 \pi a_{B}^{2} \operatorname{Ryd}^{-1} \quad f(x)=\left(1-\theta(x>1) \sqrt{x^{2}-1} / x\right)
$$

in which the local-field correction $G=0.5 q /\left(q^{2}+k_{F}^{2}\right)^{1 / 2}$, and the bare Coulomb interaction $v(q)=2 \pi e^{2} / q$ are used. The big effect of the Debye-Waller factor reflects the large vibrations in quantum systems. An example of this is reflected in the quantum Lindemann ratio, which is approximately three times larger than the classical value. In the longwavelength limit $\chi(q) \rightarrow 1 / v(q)=q r_{s}^{2} a_{B} / 2$. This is identical with the solid result, as we expect from physical considerations. That the long-wavelength limit is not a sensitive test of the nature of the ground state was previously pointed out in the study of the half-filled Landau level where essentially the same result is obtained $[7,8]$.

To study the transverse response we consider a driving force with momentum $p=(q, K)$ where the reciprocal lattice vector is $K=4 \pi / \sqrt{3} a$ for a triangular lattice. The driving energy becomes

$$
\sum_{j} v_{q} \sin \left(q x_{j}+K y_{j}\right)=v_{q}\left(\sum_{j} \sin \left(q x_{j 0}\right)+q\left(\delta x_{q}+\delta x_{-q}\right) / 2+K\left(\delta y_{q}+\delta y_{-q}\right) / 2\right)
$$


where $\delta y_{q}=N^{-0.5} \sum_{j} \delta y_{j} \exp \left(\mathrm{i} q x_{j 0}\right.$ ). For $K \gg q$, the driving force couples predominantly to the shear. The same trick is often used in neutron scattering to study transverse phonons. The analytic results for this response function are shown in figure 2 . Whereas the longitudinal response approaches zero as $q \rightarrow 0$, here the response function diverges at small momentum transfers. Also shown in figure 2 is the fluid response function. The fluid and solid responses are now very different, as expected.

An independent estimate of $\chi$ can be obtained from the energy change (1) in a fixednode Monte Carlo calculation with an energy that includes the external periodic potential $v_{\text {ext }}$. The calculation of the static response function of the fluid has recently been carried out with this idea [12].

In a variational calculation, one starts with a trial wavefunction $\Psi$ and calculates the expectation value of the Hamiltonian $\langle\Psi|H| \Psi\rangle$ with a Monte Carlo method. In the fixednode calculation $[9,10]$, one starts with the trial wavefunction as an initial state, then solves the time-dependent Schrödinger equation assuming that the position of the node of the wavefunction remains unchanged. The starting point in these calculations requires trial wavefunctions for the system. A previous Monte Carlo calculation for the undistorted system used a wavefunction $\Psi$, which was a product $(\Psi=D J)$ of a Slater determinant $D(r)$ and a Jastrow factor $J=\exp \left[-\sum_{i<j} u_{s}\left(r_{t j}\right)\right] ; D(r)$ is a determinant of Gaussian orbitals $\exp \left[-C(r-R)^{2}\right]$ localized at regular lattice sites $R$. The Fourier transform of $u_{s}$ is

$$
2 \hat{u}_{s}(k)=-1-4 C / k^{2}+\left(1+8 C / k^{2}+4 m v(k) / \hbar^{2} k^{2}\right)^{0.5} .
$$

A natural choice for the trial wavefunction in the presence of $v_{\text {ext }}$ corresponds to a product of the Jastrow factor of the pure system and a Slater determinant formed from Gaussians $\exp \left[-C\left(r-r_{j}\right)^{2}\right]$ located on lattice sites with different amounts of periodic distortion $\delta r_{j}=-\alpha_{q} v_{q} \cos \left(q \cdot r_{j 0}\right)$. Note that there is a sign and phase change between the driving force and the lattice distortion. For a given driving force, we have carried out calculations with different constants $\alpha$ so that a minimum in energy is obtained. Near the extremum, the energy does not change much as the parameter $\alpha$ is changed; $\alpha$ is determined separately for each value of $q$. If $\alpha$ were not determined correctly, we would see a large fluctuation in $\chi(q)$ as $q$ is changed. We have also tested for the validity of the linear response limit by calculating the energy changes for different driving forces. The errors from the zero-field extrapolation are less than two per cent. Our result is mostly carried out for 56 particles. Just as in previous calculations $[9,12]$, we find that the response function changes by less than five per cent as the system size is changed from 56 particles to 120 particles.

The numerical results for the longitudinal response function for different densities are shown by the open squares in figure 1 . The largest statistical error occurs at the smallest $q$ and is about 15 per cent on the average. For $q a \leqslant 6$ there is good agreement with the analytic results with the Debye-Waller factor described previously. Even though the harmonic results describe the phonon frequencies quite well, the response function is much smaller than the harmonic results except at small momentum transfers. At large momentum transfers the numerical results become bigger than the phonon contribution.

The numerical results for the transverse response function $\chi(q, K)$ for different densities are shown by the open squares in figure 2 . The Monte Carlo results are now larger than the contributions from the phonons. We think this discrepancy is real. The agreement between the numerical and the analytic results for the longitudinal response function at intermediate momentum transfers suggests that the program is correct. In addition the difference $\Delta \chi$ 

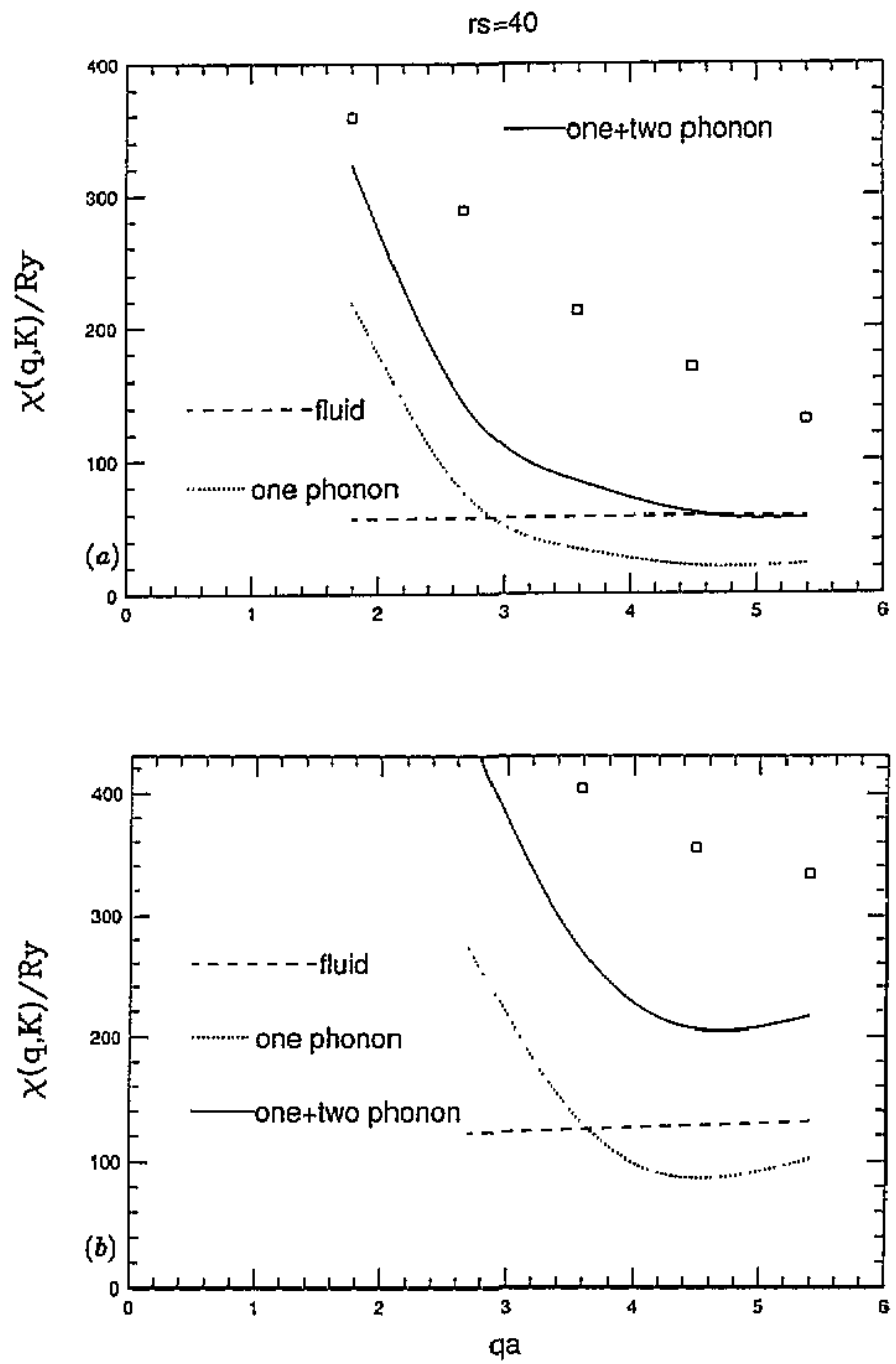

Figure 2. The 'transverse' response function $\chi(q, X)$ in units of $\mathrm{Ryd}^{-1}$ as a function of the wave vector times the lattice constant. The Monte Carlo results are indicated by the open squares. The analytic results for the one-phonon contribution with the Debye-Waller factor, the sum of the one- and two-phonon contributions and the fluid in the Hubbard approximation are shown by the dotted, full and broken curves. Different densities for $r_{s}=40$ and 75 are shown in $(a)$ and $(b)$.

cannot be due to a poor choice of the initial trial wavefunction. $\Delta \chi$ depends on $\Delta E$, the difference between the ground-state energy $E_{0}$ and the energy of the distorted state $E_{q}$. The accuracy of $E_{0}$ (not a function of $q$ ) has been tested previously [2,4]. If the initial trial wavefunction for the distorted system were not optimal, $|\Delta E|$ and $|\Delta \chi|$ would be even bigger when a better trial wavefunction was used. In addition, since $\Delta \chi$ is comparable to $\chi$, the difference cannot be accounted for by a less than 10 per cent change in phonon frequency, which should affect $\chi$ for all values of $q$. Finally, inclusion of higher phonon terms does not seem to change the shape of the $\chi$ as a function of $q$ and thus is not likely to improve the agreement with the MC results. The difference of the response functions may 
be due to inadequacies of the anharmonic calculation. It can also be due to contributions from defect waves. As we discussed in (2), different elementary excitations $|n\rangle$ contribute to the density response function. Elementary excitations such as dislocation waves [5] will provide a contribution to the response function.

In figures 1 and 2, the solid longitudinal response function is larger than the fluid response function. This situation seems to be reversed at small $r_{s}$. We have compared the phonon contribution to the density response function with that of the fluid in the Hubbard approximation. At small momentum transfers, the fluid and the solid longitudinal response functions are identical. At large momentum transfers, the fluid response is larger than that of the solid at small $r_{s}$; at large $r_{s}$, those of the solid become bigger. The solid transverse response function is always much bigger than those of the fluid.

So far we have only discussed the response function in zero magnetic field. The phonon contribution to $\chi$ in a finite field can be calculated analytically [13-15]. The result is identical in form to the zero-field case. The denominator depends not on the magnetophonon frequency but on the frequency in zero field! Now the Debye-Wailer factor depends on the filling factor $\nu$. At $v=0.2$ in the high-field limit $\left[\left\langle(\delta r)^{2}\right\rangle\right]^{1 / 2} / a$ is approximately 0.25 , comparable to the Lindemann ratio at $r_{s}=40$ in zero field [4]. We thus expect the response function at $v=0.2$ to be similar to that shown in figure 1 for $r_{s}=40$.

In summary, we studied the solid static response function with analytic and quantum Monte Carlo techniques. At intermediate momentum transfers, the longitudinal response is well approximated by the phonon contribution. For other situations, the Monte Carlo result is larger, consistent with the physical picture of additional contributions due to defect waves.

\section{Acknowledgments}

This work is supported in part by NATO grant No CRG920487. STC is grateful for the hospitality of the Physics Department at Bilkent University where part of this work was carried out.

\section{References}

[I] Willett R L, Störmer H L, Tsui D C, Pfeiffer L N, West K W and Baldwin K W 1988 Phys. Rey. B 387881 Andrei E Y, Deville G, Glattli D C and Williams F I B 1988 Phys. Rev. Lett. 602765

[2] Chui S T (ed) 1994 Physics of the 2D Quantum Electron Solid (Cambridge, MA: International)

[3] Pudalov V M, D'Iorio M, Kravchenko S V and Campbell J W 1993 Phys. Rev, Lett. 701866

[4] Esfarjani K and Chui S T $1991 \mathrm{~J}$. Phys.: Condens. Matter 35825

[5] Esfarjani K, Chui S T and Qiu X 1992 Phys. Rev. B 464638

[6] Bonsall L and Maradudin A A 1977 Phys. Rev. B 151959

[7] Chui S T 1993 Phys. Rev. B 4817343

[8] Halperin B I et al 1993 Phys. Rev. B 477312

[9] Tanatar B and Ceperley D 1989 Phys. Rev. B 395005

[10] Ceperley D 1978 Phys. Rev. B 183126

[11] Jonson M 1976 J. Phys. C: Solid State Phys. 93055

[12] Moroni S, Ceperley D M and Senatore G 1992 Phys. Rev. Lett. 691837

[13] Fisher D S 1982 Phys. Rev. B 265009

[14] Fukuyama H 1980 J. Phys. Soc. Japan 481841

[15] Esfarjani K and Chui S T 1991 Solid State Commun. 79387 